circumcision in Africa as the solution to the terrible scourge of HIV/AIDS.

\author{
Robert Clover Johnson \\ Gallaudet Research Institute \\ Gallaudet University \\ Washington, DC 20002 \\ rcloverjohnson@verizon.net
}

1. Bateman C. Millions of 'snips' will bolster our health system. S Afr Med J 2009; 99: 840-844.

\section{Mental health services for HIV/AIDS patients versus ARV services for men- tally ill HIV/AIDS patients}

To the Editor: I fully support the call made by Andersen and Seedat, ${ }^{1}$ and would like to add another dimension to the challenges posed by HIV/psychiatric co-morbidity. Apart from the psychiatric complications caused by the infection, an increasing number of - especially - the young adult psychiatric population, with chronic disorders such as schizophrenia and bipolar disorder, present with co-morbid HIV infection. Owing to the adverse socio-economic backgrounds of most public sector patients, combined with the increased socioeconomic vulnerability of chronic psychiatric patients, the HIV-positive chronic mental health care user (MHCU) is the most disadvantaged of the disadvantaged! The understandably rigorous prerequisites for ARV therapy hinder access by MHCUs to ARV treatment: they may be homeless, rejected by their families, or lead nomadic lives owing to their mental condition. These factors make identification of a 'buddy' a major challenge. Their mental condition may pose limitations on their ability to engage in the mandatory educational programme preceding commencement of treatment, or cognitive impairment from the mental illness and/or the HIV infection may diminish their capacity to give informed consent.

Finally, those with an 'organic' psychosis or HIV dementia may respond suboptimally to standard psychotropic interventions, yet be unable to access the very medication that could assist in their recovery. Special provision needs to be made in the ARV roll-out policy to cater for these patients, who may engage in risky sexual behaviour because of illness-related impairment in judgement, or themselves be 'soft' targets for sexual assault. While some may argue that a 'special' policy for MHCUs adds to discrimination and stigma, I believe that such 'discrimination' can better serve the interests of this vulnerable clinical population. Policy should facilitate MHCU access to ARV treatment, taking into consideration the challenges posed by mental illness on the consent and participation process, and make provision for active community-based worker support to facilitate adherence to both psychotropic and ARV treatment. Dedicated psychosocial support for these patients at clinic and community level, and perhaps offering a 'combined' clinic where their unique needs and challenges can be addressed, are avenues worthy of exploration.

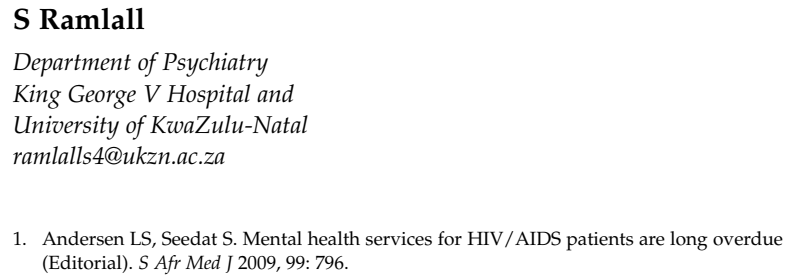

Lena Andersen replies: There is no question but that mental health services for HIV/AIDS patients are urgently needed. Recognising this is the easiest part. Developing and implementing services that cater to the entire spectrum of psychiatric needs is an enormous and complex undertaking that will require the constructive input and teamwork of many people, departments and organisations. Ramlall highlights an important issue that needs to be considered in the roll-out process. I encourage the mental health community to continue this dialogue in order to identify and address all the relevant dimensions of the problem. 